\title{
Association of SUDOSCAN Values with Vibration Perception Threshold in Chinese Patients with Type 2 Diabetes Mellitus
}

\author{
Xiaoming Zhu, ${ }^{1}$ Fei Mao, ${ }^{1}$ Siying Liu, ${ }^{1}$ Hangping Zheng, ${ }^{1}$ Bin $\mathrm{Lu},{ }^{1}$ and Yiming $\mathrm{Li}^{1,2}$ \\ ${ }^{1}$ Department of Endocrinology and Metabolism, Huashan Hospital, Fudan University, Shanghai, China \\ ${ }^{2}$ Department of Endocrinology and Metabolism, Jing'an District Center Hospital of Shanghai, Shanghai, China
}

Correspondence should be addressed to Bin Lu; binlu@fudan.edu.cn and Yiming Li; yimingli@fudan.edu.cn

Received 30 November 2016; Revised 5 April 2017; Accepted 24 April 2017; Published 20 July 2017

Academic Editor: Michael Horowitz

Copyright (C) 2017 Xiaoming Zhu et al. This is an open access article distributed under the Creative Commons Attribution License, which permits unrestricted use, distribution, and reproduction in any medium, provided the original work is properly cited.

\begin{abstract}
Aims/Introduction. SUDOSCAN has been proved to be an efficient method in detecting diabetic microvascular complications. In this study, we determine to detect the possible relationship between vibration perception threshold (VPT) and cardiac autonomic neuropathy (CAN) values produced by SUDOSCAN. Materials and Methods. A total of 920 Chinese patients with T2DM were enrolled in the study. Spearman correlation analysis and multivariate regression analysis were performed to determine the relation between CAN and VPT values. Mean VPT values across the CAN value tertiles were analyzed stratified by HbA1c status. Results. In the study, we discovered a relatively high correlation between CAN value and both VPT values from dorsal feet and toes. Multivariate regression analyses also showed a significant relation between VPT and CAN values after adjusting all covariates. The mean value of VPT decreased across the SUDOSCAN-CAN value quartiles in both groups, and it was higher in patients with $\mathrm{HbA1C}>9 \%$ than in patients with $\mathrm{HbA1C}<9 \%$ across all quartiles of the SUDOSCAN-CAN except for the VPT mean in the low quartile of the SUDOSCAN-CAN value. Conclusions. All these results suggested that SUDOSCAN-CAN result was associated with VPT value which indicated a probable link between VPT value and cardiovascular autonomic dysfunction.
\end{abstract}

\section{Introduction}

It is well known that DPN affects a large proportion of diabetic patients $(\sim 30 \%$ of hospital clinic populations and up to $22 \%$ of nonhospitalized patients) [1]. Vibration perception threshold (VPT) as a traditional method can be used to easily and accurately identify diabetic peripheral neuropathy (DPN) in everyday clinic $[1,2]$. The sensitivity of VPT measures for predicting diabetic neuropathy and related complications reportedly ranges from 77.3 to $100.0 \%$; the specificity ranges from 72.8 to $81.0 \%$ [1]. It could reflect impairment of large nerve fibers and provide evidence for diagnosis of DPN [3]. Despite for diabetic neuropathy, a previous study has demonstrated that vibration perception threshold was one of the independent risk factors for diabetic retinopathy $(\beta=0.180, P=0.015)$ [4]. Receiver operating characteristic (ROC) analysis also revealed that VPT value higher than $18 \mathrm{~V}$ was the optimal cut point for reflecting high risk of sight-threatening diabetic retinopathy (odds ratio $=4.20,95 \%$ confidence interval $=2.67-6.59$ ) [4]. Also, diabetes complications including nephropathy, retinopathy, and autonomic neuropathy have all been established as risk factors for cardiovascular disease (CVD) $[1,5,6]$. These evidences all suggest for a possible correlation between VPT and diabetic cardiovascular diseases.

SUDOSCAN (Impeto Medical, Paris, France) is a noninvasive device for the assessment of sudomotor function through evaluation of sweat gland secretory function [7]. As indicated in previous studies, SUDOSCAN can provide a risk factor score termed as CAN value to forecast the possible risks of developing cardiac autonomic neuropathy in T2DM patients [7-9]. The efficiency of CAN value detected and calculated by SUDOSCAN has been testified in several studies compared with Ewing test and HRV results.

However, there was little information about the certain relationship between VPT and diabetic cardiovascular dis- 
ease in Chinese population [5]. Since none of the previous study has focused on the possible relationship between VPT and SUDOSCAN-CAN values [8], in this study, we aimed to study the possible relationship between VPT and CAN values in T2DM patients.

\section{Subjects and Methods}

2.1. Subjects. The study was conducted in Huashan Hospital, Shanghai, from June 2014 to June 2016. The ethics committee of Huashan Hospital approved the study. Written consents were obtained from patients enrolled in the study. Patients diagnosed with type 2 diabetes between 18 and 80 years of age were continually enrolled in the study. Exclusion criteria has been described in our previous study [10] including T1DM patients, special types of diabetes, those under treatment with drugs that could have an effect on the sympathetic system such as beta blockers and antineoplastic drugs, implantation of electrical implantable devices, history of seizures or epilepsy, lumbar sciatic nerve lesion, severe varices of the lower limbs, and other metabolic diseases including thyroid disease or vitamin B12 deficiency.

2.2. Physical Examination. One trained nurse examined all the patients and recorded the results. Basic physical characteristics were recorded including height, weight, and waist and hip circumference measured by using standard methods. Body mass index (BMI) and waist hip ratio (WHR) were calculated. Blood pressure was recorded in the supine position after 5 minutes of rest. History of smoking and drinking were both recorded according to the description of the patients. Medical history of diabetes was recorded completely for each patient.

2.3. Laboratory Examination. All patients underwent $\mathrm{HbAlc}$ and renal function test. Blood samples were collected for $\mathrm{HbAlc}$ and renal function test (including serum creatinine, BUN, and uric acid) after at least 8 hours of fasting. HbAlc was determined by high-pressure liquid chromatography. Glomerular filtration rate (GFR) was calculated by Chronic Kidney Disease Epidemiology Collaboration (CKD-EPI) equation as following formula: estimated glomerular filtration rate $(\mathrm{eGFR})\left(\mathrm{mL} / \mathrm{min} / 1.73 \mathrm{~m}^{2}\right)=141 \times[\mathrm{SCR} / \mathrm{ka}] \times$ $[\mathrm{SCR} / k-1.209] \times[0.993$ age $] \times[1.018$ if female $] \quad(k$ is 0.7 for females and 0.9 for males; $a$ is -0.329 for females and -0.411 for males) [11].

2.4. Vibration Perception Threshold (VPT) Test. Vibration perception threshold was measured by the same technician by using a neuro-thesiometer (BioThesiometer; Bio-Medical Instrument Co., Newbury Ohio) [12]. Before testing, skin temperature of each patient was examined by a nurse. Then, the stimulus of neuro-thesiometer was applied to the great toe with the probe balanced vertically on the pulp of the toe on each side [13]. Patients were requested to indicate when vibration sensation was first perceived. Stimulus strength was gradually increased from null intensity to a value in voltage at which the subject first detected vibration. The whole testing procedure was carried out with the subject's eyes closed. Both feet were tested three times in a random order, and the VPT for each foot was determined as the average value of the three measurements calculated in volts. A "null stimulus" trial was added before the testing to ensure the subject's adherence and understanding. The whole testing generally required less than $3 \mathrm{~min}[2-4,13]$.

2.5. SUDOSCAN Test Procedure. The SUDOSCAN device is composed of two sets of electrodes for the feet and hands, both of which are connected to a computer for recording and data analysis. The procedure of measurement was described in previously published studies in detail. The device can measure electrochemical skin conductance (ESC) values expressed in micro-Siemens $(\mu \mathrm{S})$ for the hands and the feet (both right and left sides). The machine also had built-in algorithms which integrate electrochemical skin conductance with age, height, weight, and glycosylated hemoglobin level to produce a score that estimates current risks of diabetic cardiac autonomic neuropathy (SUDOSCAN-CAN value) [14-16].

The SUDOSCAN detection was accepted by all the subjects without any complaint of discomfort, and no safety events were ever reported as described in early studies [10].

2.6. Statistical Analysis. Results of continuously measured characteristics were expressed as median and full range; distributions of categorical variables are expressed as percentages and absolute numbers. SUDOSCAN-CAN scores were associated with VPT by multivariable median regression models adjusted for age, gender, body mass index (BMI), smoking status, alcohol consumption, and SBP. In all analyses, fractional polynomials were applied to explore and graph nonlinear associations. The doseresponse relation was found using fractional polynomials up to degree 2 with all possible combinations of powers selected from the set $(-2,-1,-0.5,0,0.5,1,2$, and 3 ) and compared using the log likelihood to determine the best-fitting model. For all multivariable analyses, a $P$ value of $<0.05$ was considered as statistically significant. All analyses were performed using Stata v11.1 (Stata Corp., College Station, TX).

2.7. Results. A total of 920 patients were enrolled in the study and further classified into three groups according to tertiles of SUDOSCAN-CAN values $(33 \%, 66 \%)$. Skin temperature of each patient examined was normal. Clinical feature was summarized in Table 1. High tertile group patients had significant higher age, longer duration of diabetes, higher BMI level, higher systolic blood pressure, and higher HbAlc level among three groups $(P<0.01)$. GFR level of high tertile group also demonstrated a significant lower level (67.8 (23.0-125.0), $P<0.0001)$ among all groups.

An association was detected between SUDOSCAN-CAN values with VPT values of both toes (left toe, $r=0.405$; right toe, $r=0.414, P$ value $<0.0001)$ and both dorsal feet (left dorsal foot, $r=0.383$; right dorsal foot, $r=0.396$, 
TABLE 1: Baseline characteristics of 920 T2DM patients stratified by SUDOSCAN-CAN value.

\begin{tabular}{|c|c|c|c|c|}
\hline & High tertile $(n=314)$ & Media tertile $(n=301)$ & Low tertile $(n=305)$ & $P$ \\
\hline Age, years & $68.0(30.0-93.0)$ & $62.0(19.0-86.0)$ & $52.0(21.0-72.0)$ & $0.000^{\mathrm{a} * *}$ \\
\hline Gender, M\% & $171(54.5 \%)$ & $187(62.1 \%)$ & $188(61.6 \%)$ & $0.094^{\mathrm{b}}$ \\
\hline Duration of disease, months & $10.0(0.0-41.0)$ & $8.5(0.0-40.0)$ & $5.0(0.0-31.0)$ & $0.000^{\mathrm{a} * *}$ \\
\hline Family history of diabetes, $n, \%$ & $60.0(21.1 \%)$ & $69.0(26.3 \%)$ & $78.0(27.5 \%)$ & $0.173^{\mathrm{b}}$ \\
\hline Smoking, $n, \%$ & $127.0(40.8 \%)$ & $144.0(48.3 \%)$ & $134.0(44.2 \%)$ & $0.177^{\mathrm{b}}$ \\
\hline Drinking, $n, \%$ & $20.0(7.0 \%)$ & $50.0(18.9 \%)$ & $54.0(19.1 \%)$ & $0.000^{\mathrm{b} * *}$ \\
\hline $\mathrm{SBP}, \mathrm{mmHg}$ & $130.0(105.0-210.0)$ & $130.0(98.0-180.0)$ & $120.0(90.0-180.0)$ & $0.000^{\mathrm{a} * *}$ \\
\hline $\mathrm{DBP}, \mathrm{mmHg}$ & $80.0(50.0-102.0)$ & $80.0(60.0-110.0)$ & $80.0(51.0-110.0)$ & $0.274^{\mathrm{a}}$ \\
\hline BMI, $\mathrm{kg} / \mathrm{m}^{2}$ & $25.0(17.0-41.0)$ & $24.0(15.0-40.0)$ & $23.0(17.0-35.0)$ & $0.000^{\mathrm{a} * *}$ \\
\hline Waist circumference, $\mathrm{cm}$ & $93.0(69.0-160.0)$ & $90.0(64.0-129.0)$ & $87.0(62.0-121.0)$ & $0.008^{\mathrm{a} * *}$ \\
\hline WHR & $13.7(1.8-49.9)$ & $10.4(3.0-49.2)$ & $7.3(2.0-49.6)$ & $0.000^{\mathrm{a} * *}$ \\
\hline $\mathrm{GFR}, \mathrm{mL} / \mathrm{min} / 1.73 \mathrm{~m}^{2}$ & $67.8(23.0-125.0)$ & $84.6(42.0-133.0)$ & $83.7(49.0-125.0)$ & $0.000^{\mathrm{a} * *}$ \\
\hline $\mathrm{HbA} 1 \mathrm{C}, \%$ & $8.8(6.0-18.0)$ & $7.5(6.0-13.0)$ & $6.7(5.0-11.0)$ & $0.000^{\mathrm{a} * *}$ \\
\hline Mean VPT value (V) & $13.7(1.8-49.8)$ & $10.4(3.0-49.2)$ & $7.3(2.0-49.5)$ & $0.000^{\mathrm{a} * *}$ \\
\hline
\end{tabular}

SBP: systolic blood pressure; BMI: body mass index; WHR: waist hip ratio; HbA1C: hemoglobin A1C; GFR: glomerular filtration rate; VPT: vibration perception threshold; ESC: electrochemical skin conductance; ${ }^{\mathrm{a}} \mathrm{P}$ for Kruskal-Wallis $\mathrm{H}$ test; ${ }^{\mathrm{b}}$ chi-square tests; ${ }^{* *} P$ value $<0.01$.

TABLE 2: Spearman's correlation coefficients between feet ESC values, CAN value, and indices of VPT (toes and dorsal feet).

\begin{tabular}{lcccc}
\hline & \multicolumn{2}{c}{ Toe } & \multicolumn{2}{c}{ Dorsal foot } \\
& Left toe & Right toe & $\begin{array}{c}\text { Left dorsal } \\
\text { foot }\end{array}$ & $\begin{array}{c}\text { Right dorsal } \\
\text { foot }\end{array}$ \\
\hline CAN & $0.405^{* * *}$ & $0.414^{* * *}$ & $0.383^{* * *}$ & $0.396^{* * *}$ \\
Left foot ESC & $-0.160^{* * *}$ & $-0.202^{* * *}$ & $-0.149^{* * *}$ & $-0.150^{* * *}$ \\
Right foot ESC & $-0.153^{* * *}$ & $-0.207^{* * *}$ & $-0.140^{* * *}$ & $-0.137^{* * *}$ \\
\hline
\end{tabular}

CAN: cardiac autonomic neuropathy; VPT: vibration perception threshold; ESC: electrochemical skin conductance; ${ }^{* * *} P<0.0001$.

$P$ value $<0.0001)$, respectively. Lower ESC values of SUDOSCAN were also significantly associated with increasing VPT values $(P<0.0001)$ (Table 2$)$.

We determined to further explore the association between SUDOSCAN-CAN and VPT values in different HbA1C levels. Mean VPT value was performed across the quartiles of the SUDOSCAN-CAN value with patients stratified by $\mathrm{HbA1C}$ level at the cutoff of $9 \%$ as shown in Figure 1. The mean value of VPT decreased across the SUDOSCANCAN value quartiles in both groups $(P$ for trend $<0.05$ for all). And furthermore, the mean value of VPT was higher in patients with $\mathrm{HbA1C}>9 \%$ than in patients with $\mathrm{HbA} 1 \mathrm{C}<9 \%$ across all quartiles of the SUDOSCAN-CAN value $(P<0.05$ for all), except for the VPT mean in the low quartile of the SUDOSCAN-CAN value.

Multivariate regression analyses of the association between SUDOSCAN values and VPT indices are shown in Table 3. In the unadjusted regression models, the SUDOSCAN-CAN value was positively associated with VPT values (including left toe, beta $=0.58, P<0.0001$; right toe, beta $=0.66, P<0.0001$; left dorsal foot, beta $=0.60$, $P<0.0001$; right dorsal foot, beta $=0.54, P<0.0001)$. Feet

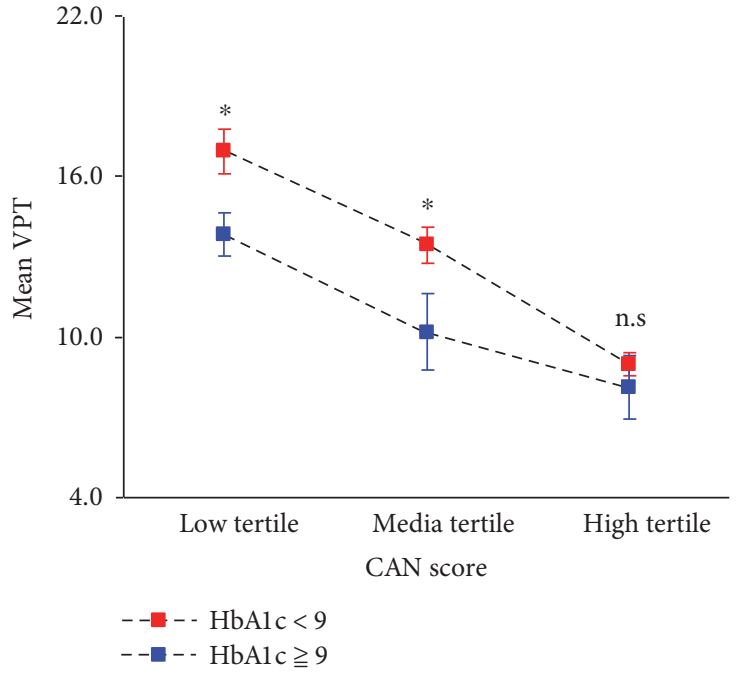

FIgURE 1: Mean VPT values across the CAN value tertiles, stratified by $\mathrm{HbA1c}$ status. Error bars represent $95 \% \mathrm{CI}$ of the mean. n.s: not significant. ${ }^{*} P$ value $<0.05$.

ESC values of both sides were all negatively associated with VPT values $(P<0.0001)$. After adjustment for all covariates including age, gender, course of disease, SBP, drinking, smoking, and BMI, the CAN value remained positively associated with VPT values (including left toe, beta $=0.19, P<0.01$; right toe, beta $=0.26, P<0.0001$; left dorsal foot, beta $=0.16, P<0.01$; right dorsal foot, beta $=0.19, P<0.0001)$. Feet ESC values of both sides remained all negatively associated with VPT values $(P<0.0001)$. Adjusted regression curves showing the association between CAN value and the mean VPT value was demonstrated in Figure 2. 
TABLE 3: Multivariate regression analyses of the association between the SUDOSCAN values and VPT indices.

\begin{tabular}{lcccc}
\hline & \multicolumn{4}{c}{ VPT values } \\
& $\begin{array}{c}\text { Left toe } \\
\operatorname{Beta}(P)\end{array}$ & $\begin{array}{c}\text { Right toe } \\
\operatorname{Beta}(P)\end{array}$ & $\begin{array}{c}\text { Left dorsal } \\
\text { foot } \\
\operatorname{Beta}(P)\end{array}$ & $\begin{array}{c}\text { Right dorsal } \\
\text { foot } \\
\operatorname{Beta}(P)\end{array}$ \\
\hline CAN value & & & & \\
Unadjusted & $0.578^{* * *}$ & $0.660^{* * *}$ & $0.496^{* * *}$ & $0.543^{* * *}$ \\
Adjusted & $0.191^{* *}$ & $0.261^{* * *}$ & $0.157^{* *}$ & $0.186^{* * *}$ \\
Left foot & & & & \\
ESC value & & & & \\
Unadjusted & $-0.561^{* * *}$ & $-0.595^{* * *}$ & $-0.498^{* * *}$ & $-0.440^{* * *}$ \\
Adjusted & $-0.496^{* * *}$ & $-0.539^{* * *}$ & $-0.487^{* * *}$ & $-0.374^{* * *}$ \\
Right foot & & & & \\
ESC value & & & & \\
Unadjusted $^{\text {a }}$ & $-0.556^{* * *}$ & $-0.605^{* * *}$ & $-0.487^{* * *}$ & $-0.432^{* * *}$ \\
Adjusted $^{\mathrm{a}}$ & $-0.478^{* * *}$ & $-0.530^{* * *}$ & $-0.435^{* * *}$ & $-0.324^{* * *}$ \\
\hline
\end{tabular}

${ }^{a}$ Model is adjusted for all covariates including age, gender, course of disease, SBP, drinking, smoking, and BMI. CAN: cardiac autonomic neuropathy; VPT: vibration perception threshold; ESC: electrochemical skin conductance; ${ }^{* * *} P<0.0001 ;{ }^{* *} P<0.01$.

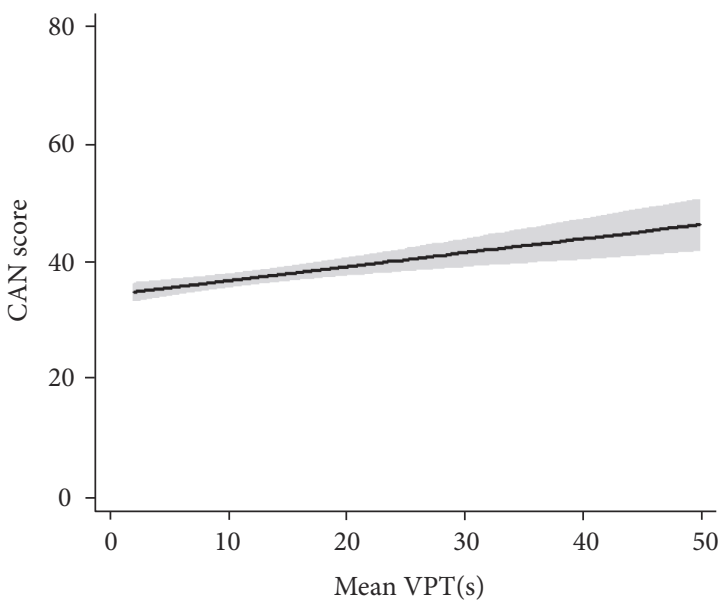

FIGURE 2: Adjusted regression curves showing the association between CAN score and the mean VPT value. Shaded area represents confidence intervals (CIs).

\section{Discussion}

This is the very first time to study the association between VPT and CAN values produced by SUDOSCAN device in Chinese patients with T2DM. A total number of 920 T2DM patients were enrolled in the study. VPT and SUDOSCAN tests were all performed, and results were recorded and analyzed. In year 2011, Gin et al. [17] once measured VPT and ESC values in 142 European diabetic patients. ESC measurements in the feet of patients showed that the correlation between VPT and ESC was $-0.45(P<0.0001)$. Similar study was conducted in year 2012 [7] which showed lower ESC at feet was significantly associated with increasing VPT by biothesiometry $(P<0.01)$. In the study, a total number of 265 T2DM patients underwent both Ewing test which consists of four different parts including $\mathrm{E} / \mathrm{I}$ ratio, 30/15 ratio, orthostatic hypotension, and Valsalva test) and SUDOSCAN test. The result showed that compared to patients with $\mathrm{ESC} \geq 40 \mu \mathrm{S}$, patients with $\mathrm{ESC}<40 \mu \mathrm{S}$ were more than four times likely to have or more CAN tests abnormal $(\mathrm{OR}=4.41(1.72-11.29))$ [7]. These results all indicated a possible relationship between ESC value and VPT value.

In our study, we analyzed the correlation between feet and hands ESC value with VPT values of toe and dorsal foot by Spearman correlation analysis, respectively. The results showed significant correlations between VPT and ESC values which was in consistent with previous studies $(P<0.001)$. Yet in the study, we discovered a relatively high correlation between CAN value with both VPT values from dorsal feet (left dorsal foot $r=0.383, P<0.001$; right dorsal foot $r=0.396, P<0.001$ ) and toes (left toe $r=0.405, P<0.001$; right toe $r=0.414, P<0.001)$. Multivariate regression analyses also showed a significant relation between VPT and CAN values after adjusting all covariates including age, gender, course of disease, SBP, drinking, smoking, and BMI (including left toe, beta $=0.19, P<0.01$; right toe, beta $=0.26$, $P<0.0001$; left dorsal foot, beta $=0.16, P<0.01$; right dorsal foot, beta $=0.19, P<0.0001)$. These all suggested that VPT value might predict CAN result since they had a high correlation. We further classified patients into three groups according to tertiles of SUDOSCAN-CAN value (33\%, $66 \%$ ). The possibility of developing cardiovascular automatic disease decreases as SUDOSCAN-CAN value increases. We found that high tertile group patients had significant higher age, longer duration of diabetes, higher BMI level, higher systolic blood pressure, higher GFR value, and higher HbAlc level among three groups. After stratified by $\mathrm{HbAlc}$ status, the mean value of VPT decreased across the SUDOSCANCAN value quartiles in both groups. And furthermore, we discovered that the mean value of VPT was higher in patients with $\mathrm{HbA1C}>9 \%$ than in patients with HbA1C $<9 \%$ across all quartiles of the SUDOSCAN-CAN value $(P<0.05$ for all), except for the VPT mean in the low quartile of the SUDOSCAN-CAN value. All the results in our study suggested a possible relationship between VPT and SUDOSCAN-CAN values which has not been reported before in other study. Since diabetic neuropathies including both peripheral and cardiovascular automatic neuropathy share some common underlying pathological mechanisms, the results could be explained. Strengths of the current study include a large-scale, population-based design by using the SUDOSCAN testing device. The study further suggested us look into the possible role of VPT in predicting diabetic cardiovascular diseases.

However, there were several limitations in the study that must be clarified. In the study, we simply provided the evidence of possible correlation between CAN score with VPT value in this cross-sectional study whereas we did not perform the confirmatory tests of DPN such as electromyography test. Secondly, this observational study simply gives us a hint that VPT might predict diabetic cardiovascular disease. But we still need to do a longitudinal study to validate this possibility. Thus, further tests need to be performed to confirm the results in this study. 


\section{Conclusion}

SUDOSCAN-CAN result was associated with VPT value which indicated a probable link between VPT value and cardiovascular autonomic dysfunction.
Abbreviations
CAN: Cardiac autonomic neuropathy
VPT: Vibration perception threshold
ESC: Electrochemical skin conductance
T2DM: Type 2 diabetes mellitus
DBP: Diastolic blood pressure
SBP: $\quad$ Systolic blood pressure
BMI: $\quad$ Body mass index
WHR: Waist hip ratio
HbA1C: Hemoglobin A1C
GFR: Glomerular filtration rate.

\section{Conflicts of Interest}

The authors declare no conflict of interest.

\section{Authors' Contributions}

Xiaoming Zhu and Fei Mao contributed equally to this work.

\section{Acknowledgments}

This study was supported by grants from the National Natural Science Foundation of China (81370884, to Bin Lu), the Shanghai New Excellent Youth Program (XYQ2013120, to Bin Lu) and Fudan Zhuoxue Project (to Bin Lu), the Pudong Program from Pudong Municipal Commission of Health and Family Planning (PW2014D-2, to Bin Lu), and the Shanghai Science and Technology Committee Program (14411962200, to Yiming Li).

\section{References}

[1] A. P. Garrow and A. J. Boulton, "Vibration perception threshold-a valuable assessment of neural dysfunction in people with diabetes," Diabetes/Metabolism Research and Reviews, vol. 22, no. 5, pp. 411-419, 2006.

[2] Y. Hou, S. Liu, T. Zhu et al., "Vibration perception threshold in diagnosing diabetic peripheral neuropathy by receiver operating characteristic curve," Zhong nan da Xue Xue Bao Yi Xue ban = Journal of Central South University Medical Sciences, vol. 37, no. 9, pp. 951-956, 2012.

[3] C. L. Martin, B. H. Waberski, R. Pop-Busui et al., "Vibration perception threshold as a measure of distal symmetrical peripheral neuropathy in type 1 diabetes: results from the DCCT/EDIC study," Diabetes Care, vol. 33, no. 12, pp. 2635-2641, 2010.

[4] J. Shen, Y. Hu, F. Liu et al., "Vibration perception threshold for sight-threatening retinopathy screening in type 2 diabetic outpatients," Diabetes/Metabolism Research and Reviews, vol. 29, no. 7, pp. 525-531, 2013.

[5] R. E. Maser and M. J. Lenhard, "Cardiovascular autonomic neuropathy due to diabetes mellitus: clinical manifestations, consequences, and treatment," The Journal of Clinical Endocrinology and Metabolism, vol. 90, no. 10, pp. 5896-5903, 2005.

[6] J. Elliott, S. Tesfaye, N. Chaturvedi et al., "Large-fiber dysfunction in diabetic peripheral neuropathy is predicted by cardiovascular risk factors," Diabetes Care, vol. 32, no. 10, pp. 1896-1900, 2009.

[7] C. S. Yajnik, V. V. Kantikar, A. J. Pande, and J. P. Deslypere, "Quick and simple evaluation of sudomotor function for screening of diabetic neuropathy," ISRN Endocrinology, vol. 2012, Article ID 103714, 7 pages, 2012.

[8] A. I. Vinik, M. L. Nevoret, and C. Casellini, "The new age of sudomotor function testing: a sensitive and specific biomarker for diagnosis, estimation of severity, monitoring progression, and regression in response to intervention," Frontiers in Endocrinology, vol. 6, p. 94, 2015.

[9] C. M. Casellini, H. K. Parson, M. S. Richardson, M. L. Nevoret, and A. I. Vinik, "Sudoscan, a noninvasive tool for detecting diabetic small fiber neuropathy and autonomic dysfunction," Diabetes Technology \& Therapeutics, vol. 15, no. 11, pp. 948953, 2013.

[10] F. Mao, S. Liu, X. Qiao et al., "Sudoscan is an effective screening method for asymptomatic diabetic neuropathy in Chinese type 2 diabetes mellitus patients," Journal of Diabetes Investigation, vol. 8, no. 3, pp. 363-368, 2016.

[11] A. O. Luk, W. C. Fu, X. Li et al., "The clinical utility of SUDOSCAN in chronic kidney disease in Chinese patients with type 2 diabetes," PLoS One, vol. 10, no. 11, article e0134981, 2015.

[12] T. Mete, Y. Aydin, M. Saka et al., "Comparison of efficiencies of Michigan neuropathy screening instrument, neurothesiometer, and electromyography for diagnosis of diabetic neuropathy," International Journal of Endocrinology, vol. 2013, Article ID 821745, 7 pages, 2013.

[13] M. J. Young, J. L. Breddy, A. Veves, and A. J. Boulton, "The prediction of diabetic neuropathic foot ulceration using vibration perception thresholds. A prospective study," Diabetes Care, vol. 17, no. 6, pp. 557-560, 1994.

[14] G. Muller, E. Parfentyeva, J. Olschewsky, S. R. Bornstein, and P. E. Schwarz, "Assessment of small fiber neuropathy to predict future risk of type 2 diabetes," Primary Care Diabetes, vol. 7, no. 4, pp. 269-273, 2013.

[15] B. I. Freedman, D. W. Bowden, S. C. Smith, J. Xu, and J. Divers, "Relationships between electrochemical skin conductance and kidney disease in type 2 diabetes," Journal of Diabetes and Its Complications, vol. 28, no. 1, pp. 56-60, 2014.

[16] A. G. Smith, M. Lessard, S. Reyna, M. Doudova, and J. R. Singleton, "The diagnostic utility of Sudoscan for distal symmetric peripheral neuropathy," Journal of Diabetes and Its Complications, vol. 28, no. 4, pp. 511-516, 2014.

[17] H. Gin, R. Baudoin, C. H. Raffaitin, V. Rigalleau, and C. Gonzalez, "Non-invasive and quantitative assessment of sudomotor function for peripheral diabetic neuropathy evaluation," Diabetes \& Metabolism, vol. 37, no. 6, pp. 527$532,2011$. 


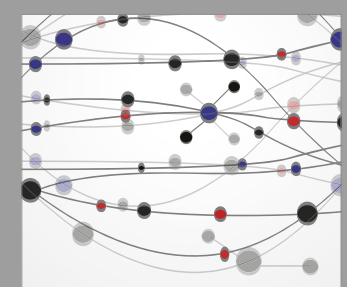

The Scientific World Journal
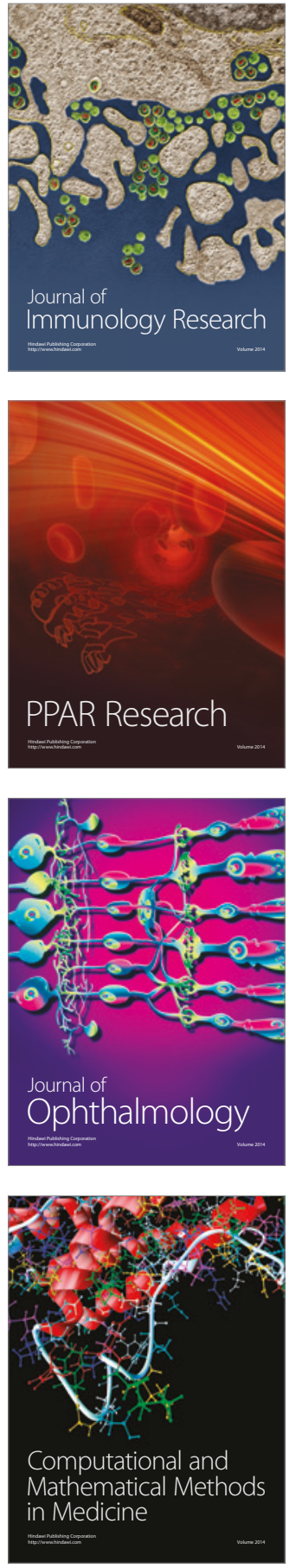

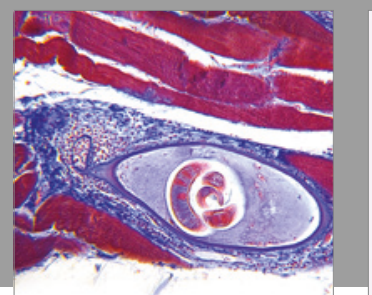

Gastroenterology Research and Practice
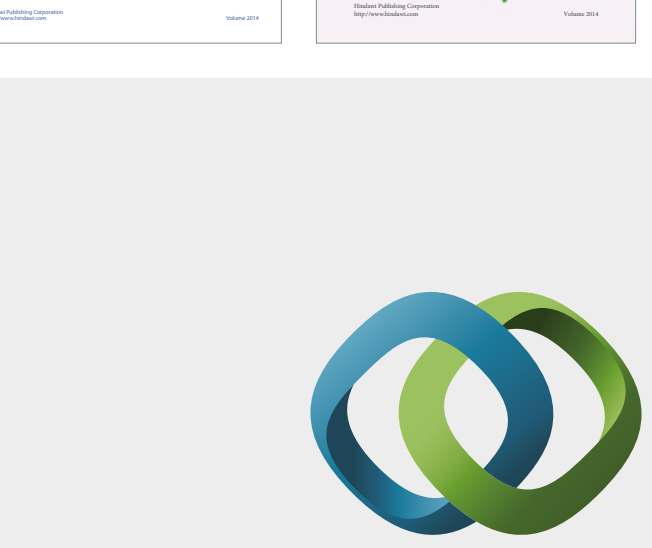

\section{Hindawi}

Submit your manuscripts at

https://www.hindawi.com
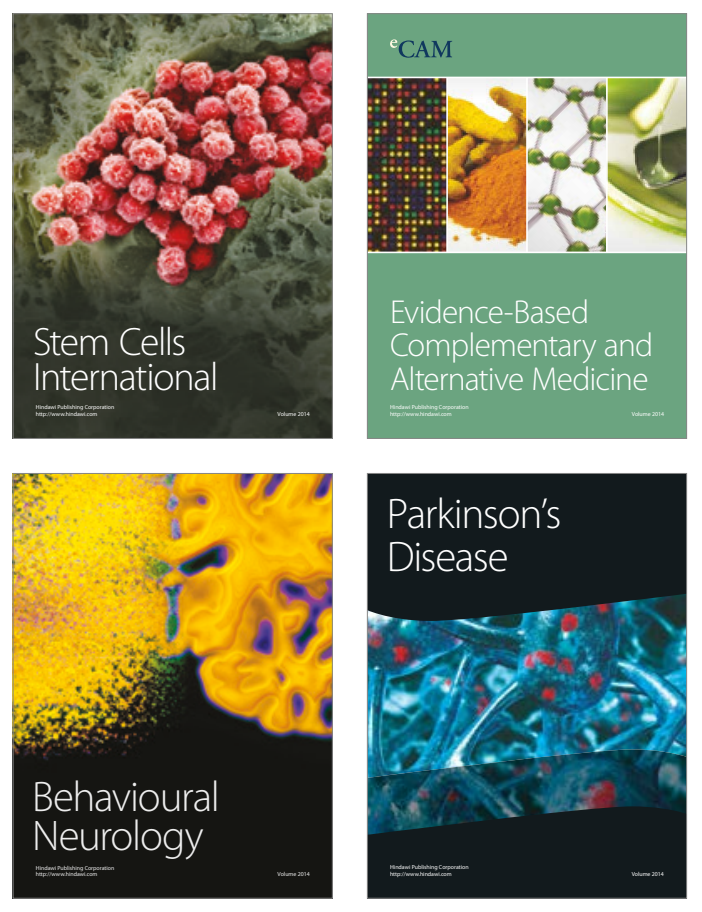
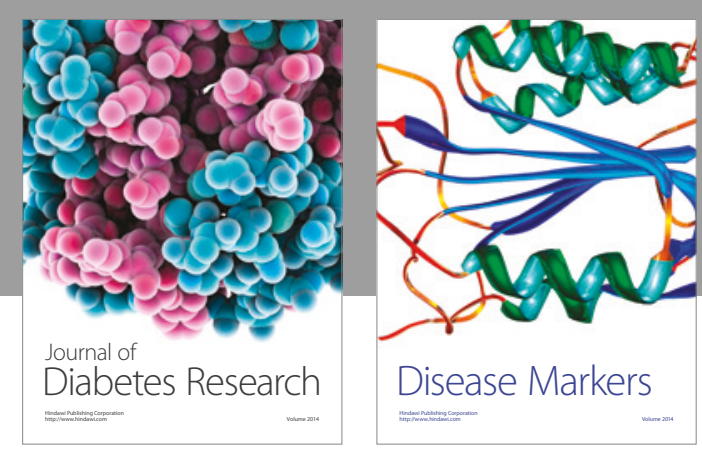

Disease Markers
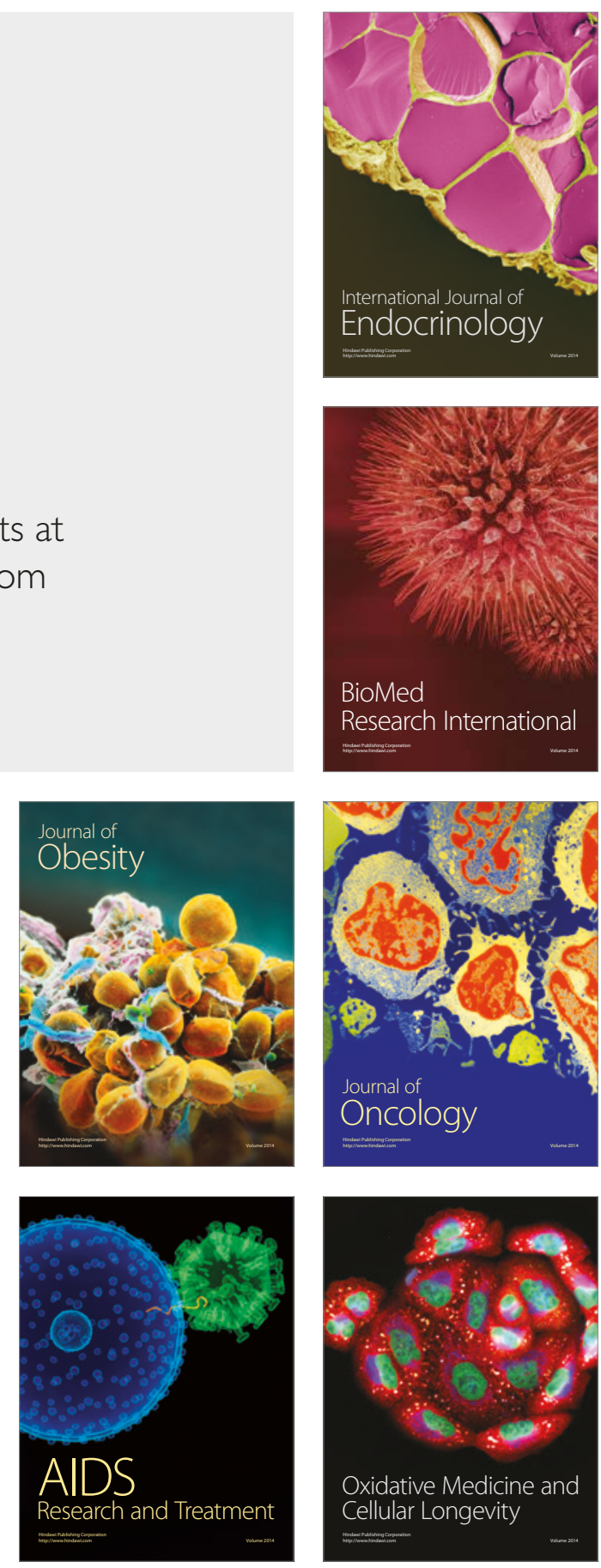